\title{
具有梯度渐变折射率的超疏水减反膜的制备及其性能
}

\author{
英桂玉，李怡雯，王红宁，陈若愚 \\ (常州大学 石油化工学院, 常州 213164)
}

摘 要: 具有梯度折射率的减反膜可以在更宽光谱波段和更大入射光角度实现减反射性能。本研究利用溶胶-凝胶 和溶剂热法分别合成实心氧化硅 $\left(\mathrm{SiO}_{2}\right)$ 、空心氧化硅 $\left(\mathrm{H}-\mathrm{SiO}_{2}\right)$ 和空心氟化镁 $\left(\mathrm{MgF}_{2}\right)$ 溶胶, 利用浸渍-提拉法在玻璃双 面镀制 $\mathrm{SiO}_{2} / \mathrm{H}-\mathrm{SiO}_{2} / \mathrm{MgF}_{2}$ 梯度折射率薄膜。结果表明, 在 380 1600 nm 波长, 镀膜基片在光垂直入射时透光率高达 $99.88 \%$, 当光以 $0^{\circ} \sim 45^{\circ}$ 入射时, 平均透光率均高于 $97.85 \%$, 即使光以 $75^{\circ}$ 入射时, 最高透光率仍达 $95.51 \%$ 。同时发 现，经十六烷基三甲氧基硅烷(HDTMOS)修饰，薄膜疏水角达到 $150.6^{\circ}$ ，显示出良好的疏水自清洁效果。

关 键 词: 梯度折射率; 全方位; 宽带; 疏水; 减反膜

中图分类号: TB34 文献标识码: A

\section{Preparation and Property of Superhydrophobic Antireflective Film with Gradient Refractive Index}

\author{
JIA Gui-Yu, LI Yi-Wen, WANG Hong-Ning, CHEN Ruo-Yu \\ (College of Petrochemical Engineering, Changzhou University, Changzhou 213164, China)
}

\begin{abstract}
Gradient refractive index antireflection film achieves antireflective performance in wider spectrum band at larger incident of angle. In this study, solid silica nanometer particles $\left(\mathrm{SiO}_{2}\right)$ sol and hollow silica nanometer particles $\left(\mathrm{H}-\mathrm{SiO}_{2}\right)$ sol were synthesized by Sol-Gel method, while hollow rod-shaped magnesium fluoride nanometer particles $\left(\mathrm{MgF}_{2}\right)$ sol was prepared by solvothermal method. Then, the $\mathrm{SiO}_{2} / \mathrm{H}-\mathrm{SiO}_{2} / \mathrm{MgF}_{2}$ gradient refractive index antireflection film was successfully coated on double sides of borosilicate glass by dip-coating method. The experimental results show that in the wavelength range of $380 \sim 1600 \mathrm{~nm}$, when light is incident vertically, the transmittance of the coated glass reaches $99.88 \%$; when the light is at the incident angle of $0^{\circ} \sim 45^{\circ}$, with the average transmittance higher than $97.85 \%$. And the light is even at the incident angle of $75^{\circ}$, its maximum transmittance is still as high as $95.51 \%$. After modified by hexadecyl trimethoxysilane (HDTMOS) the contact angle of the coated glass is up to $150.6^{\circ}$, showing good hydrophobic self-cleaning performance.
\end{abstract}

Key words: gradient refractive index; omnidirectional; broadband; hydrophobic; antireflective film

太阳光在光热或光伏组件表面的反射损失是影 响光电或光热转换效率重要因素之一 ${ }^{[1-2]}$ 。未镀减反 膜的光伏组件的表面反射损失高达 $36 \%$ 37\%, 这 大大限制了光电转换效率 ${ }^{[3]}$ 。并且, 光伏光热组件长
期在易污染的野外环境中使用。因此制备宽谱带、 全方位减反射并具有自洁功能的减反射薄膜在太阳 能利用领域具有重要意义。

当均一的单层减反膜满足最优折射率和光学厚

收稿日期：2017-12-01; 收到修改稿日期：2018-02-09

基金项目：国家自然科学基金(21571024); 江苏省研究生科研与实践创新计划(KYZZ16_0248, KYCX17_2089) National Natural Science Foundation of China (21571024); Postgraduate Research and Practice Innovation Program of Jiangsu Province (KYZZ16_0248, KYCX17_2089)

作者简介：荚桂玉(1993-), 女，硕士研究生. E-mail: jgy334317@163.com

通讯作者：陈若愚，教授. E-mail: cxdcry@163.com 
度时, 可以实现单波长最优减反射效果 ${ }^{[4]}$, 但剩余 波长反射率仍很高。而具有梯度折射率的多层薄膜 可以实现宽波段和宽角度减反射效果 ${ }^{[5-6]}$ 。Camargo 等 ${ }^{[7]}$ 通过浸渍-提拉和物理气相沉积法制备出三层 渐变折射率薄膜, 在 1600 2000 nm 波段内, 光垂直 入射的透光率高达 $99.5 \%$, 光在 $20^{\circ}$ 入射角时的透光 率只下降 $2 \%$ 。 $\mathrm{Liu}^{\text {等 }}{ }^{[8]}$ 利用化学刻蚀法构建梯度折 射率层, 在 350 1500 nm 波段内, 光入射角在 $70^{\circ}$ 时, 透光率从 $73.1 \%$ 提高到 $95.4 \%$ 。相比物理气相沉积 和化学刻蚀等方法, 溶胶-凝胶法可以实现低成本 和大面积镀膜, 更具有工业应用的优势。Yildirim 等 ${ }^{[9]}$ 利用溶胶- 凝胶法制备三层梯度折射率薄膜, 入射 角为 $60^{\circ}$ 时, 在 350 2200 $\mathrm{nm}$ 内的平均透光率比空 白基片增加了约 $8 \%$ 。构建梯度折射率减反膜需在 顶层构建低折射率膜层, 而现有镀膜材料的折射率 最低为 $1.38\left(\mathrm{MgF}_{2}\right)$, 无法满足要求。材料孔隙率越 大, 其对应的折射率越低 ${ }^{[10]}$, 若制备出空心结构减 反膜材料, 将很大程度地降低膜层折射率。

本工作利用实心氧化硅、空心氧化硅粒子和空 心棒状氟化镁三种纳米材料制备折射率逐渐减小的 三层减反射薄膜。对薄膜在 380 1600 nm 波长范围 内, $0 \sim 75^{\circ}$ 光入射角下的光学性质进行研究, 并对薄 膜的疏水情况进行了分析, 以期实现膜层的宽谱 带、全方位减反射和疏水自清洁多功能性能。

\section{1 实验方法}

\section{1 粒子溶胶的制备}

实心 $\mathrm{SiO}_{2}$ 溶胶的制 备 ${ }^{[11]}: 2.02 \mathrm{~mL}$ 氨水 $\left(\mathrm{NH}_{3} \cdot \mathrm{H}_{2} \mathrm{O}, 28 \%\right)$ 加入 $65.5 \mathrm{~mL}$ 无水乙醇 $(\mathrm{EtOH}$, $99.9 \%), 30^{\circ} \mathrm{C}$ 水浴搅拌, 逐滴加入 $6.67 \mathrm{~mL}$ 正硅酸乙 酯 (TEOS, 99\%)中, 反应 $6 \mathrm{~h}$ 后陈化 $4 \mathrm{~d}$, 除氨稀释 $\mathrm{SiO}_{2}$ 质量分数为 $1 \mathrm{wt} \%$, 记为 $\mathrm{a}$ 样。

空心 $\mathrm{SiO}_{2}$ 溶胶的制备 ${ }^{[12]}: 0.12 \mathrm{~g}$ 聚丙烯酸 (PAA, $\mathrm{Mw} \approx 5000,30 \mathrm{wt} \%$ ) 溶于 $6 \mathrm{~mL} \mathrm{NH} \cdot \mathrm{H}_{2} \mathrm{O}$ 中, 再加入 $120 \mathrm{~mL}$ EtOH 混合搅拌均匀, 每隔 $10 \mathrm{~min}$ 逐

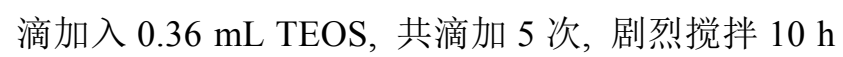
后除氨, 调整 $\mathrm{H}-\mathrm{SiO}_{2}$ 质量分数为 $2 \mathrm{wt} \%$, 记为 $\mathrm{b}$ 样。

空心棒状 $\mathrm{MgF}_{2}$ 溶胶的制备 ${ }^{[13]}: 1.73 \mathrm{~g}$ 四水合 乙酸美 $\left(\mathrm{Mg}\left(\mathrm{CH}_{3} \mathrm{COO}\right)_{2} \cdot 4 \mathrm{H}_{2} \mathrm{O}, 99 \%\right)$ 溶于 $38 \mathrm{~g}$ 无水甲 醇 $\left(\mathrm{CH}_{3} \mathrm{OH}, 99.9 \%\right.$ )中, 缓慢滴加由 $9.3 \mathrm{~g} \mathrm{CH}_{3} \mathrm{OH}$ 稀 释的 $0.645 \mathrm{~g}$ 氢氟酸 $(\mathrm{HF}, 40 \mathrm{wt} \%)$ 溶液, 常温反应 $30 \mathrm{~min}$ 后, $240^{\circ} \mathrm{C}$ 烘箱反应 $24 \mathrm{~h}$, 制得 $\mathrm{MgF}_{2}$ 溶胶, 记 为 $\mathrm{C}$ 样。

线性 $\mathrm{SiO}_{2}$ 溶胶的制备 ${ }^{[14]}$ : 将 $36 \mathrm{~g} \mathrm{H}_{2} \mathrm{O}$ 以及 $0.2 \mathrm{~g}$ 浓盐酸 $(\mathrm{HCl}, 37 \%)$ 加入到 $860 \mathrm{~g} \mathrm{EtOH}$ 中, $30^{\circ} \mathrm{C}$ 水浴
搅拌, 然后逐滴加入 $104 \mathrm{~g}$ TEOS, $6 \mathrm{~h}$ 后陈化 $4 \mathrm{~d}$, 所 得溶胶记为 $\mathrm{S}$ 样。

试剂均购自国药化学试剂有限公司, 没有进一 步处理。

\section{2 三层梯度折射率减反薄膜的制备}

以高嗍硅玻璃 $(100 \mathrm{~mm} \times 25 \mathrm{~mm} \times 3 \mathrm{~mm})$ 为基片, 清洗处理按文献[15]进行。以 $\mathrm{SiO}_{2}$ 的摩尔比分别为 $4: 6$ 和 $1: 9$ 的比例将 $S$ 样加入 $a 、 b$ 样中, 得到 $A$ 、 B 镀膜溶胶。

采用 SYDC-100 浸渍-提拉机，将玻璃基片浸入 $\mathrm{A}$ 溶胶中提拉镀制底层实心 $\mathrm{SiO}_{2}$ 薄膜, 在 $80^{\circ} \mathrm{C}$ 烘箱 中干燥 $30 \mathrm{~min}$, 再将镀好底层薄膜的基片浸入 $\mathrm{B}$ 溶 胶中提拉镀制中间层 $\mathrm{H}-\mathrm{SiO}_{2}$ 薄膜, 在 $80^{\circ} \mathrm{C}$ 烘箱中干 燥 $30 \mathrm{~min}$, 然后将镀制两层薄膜的基片置于马弗炉 $500^{\circ} \mathrm{C}$ 焙烧 $2 \mathrm{~h}$ 。最后将烧过的基片浸入 $\mathrm{C}$ 溶胶提拉 镀制顶层 $\mathrm{MgF}_{2}$ 薄膜, 在 $240^{\circ} \mathrm{C}$ 烘箱稳定膜层 $2 \mathrm{~h}$ 后 即得到 $\mathrm{SiO}_{2} / \mathrm{H}-\mathrm{SiO}_{2} / \mathrm{MgF}_{2}$ 梯度折射率薄膜。随后, 用 HDTMOS (Aladdin, $85 \%$ ) $^{[15]}$ 进行表面修饰, 室温 干燥得到疏水减反膜。

\section{3 表征}

采用日本 JEM-2100 型高分辨透射电镜 (TEM) 观察 $a 、 b 、 C$ 样中纳米粒子的形态结构。采用德国蔡司 SUPRA55 型场发射扫描电镜(FESEM)、美国 Veeco NanoMan VS 原子力显微镜(AFM)观察三层 薄膜微观表面形貌。使用椭偏仪(北京量拓科技有限 公司)建立物理模型 “Air-CAUCHY-Si” 拟合得到每 层薄膜的折射率 $n$ 。采用日本岛津 UV-1700 型紫外可见分光光度计测量在 380 1600 nm 范围内, 镀膜 基片在 $0 \sim 75^{\circ}$ 入射角的透光率。将 Glan-Thompson 偏振棱镜置于光谱测量系统, 测量相应的 $s$-和 $p$-偏 振光的透光率曲线。采用 HARKE-SPCA 标准接触 角测量仪测定镀膜基片的水接触角, 对薄膜自清洁 性能进行表征。采用德国 Erichsen 公司 291 型铅笔 硬度测试仪对薄膜耐磨性能进行评价; 采用 WS-92 划痕仪测定薄膜与基底的结合力。

\section{2 结果与讨论}

\section{$2.1 \mathrm{SiO}_{2} /{\mathrm{H}-\mathrm{SiO}_{2}} / \mathrm{MgF}_{2}$ 梯度折射率薄膜的表 面形貌}

$\mathrm{SiO}_{2}$ 和 $\mathrm{H}-\mathrm{SiO}_{2}$ 纳米粒子以及 $\mathrm{MgF}_{2}$ 纳米棒的高 分辨透射电镜照片见图 $1 。 \mathrm{SiO}_{2}$ 粒子的平均粒径约 $15 \mathrm{~nm}$, 粒子近似球状(图 1(a)); $\mathrm{H}-\mathrm{SiO}_{2}$ 粒子的平均 粒径约 $65 \mathrm{~nm}$, 其壁厚约 $17 \mathrm{~nm}$, 粒子均一性良好 (图 1(b)); 空心棒状 $\mathrm{MgF}_{2}$ 的平均棒长约 $160 \mathrm{~nm}$, 平 

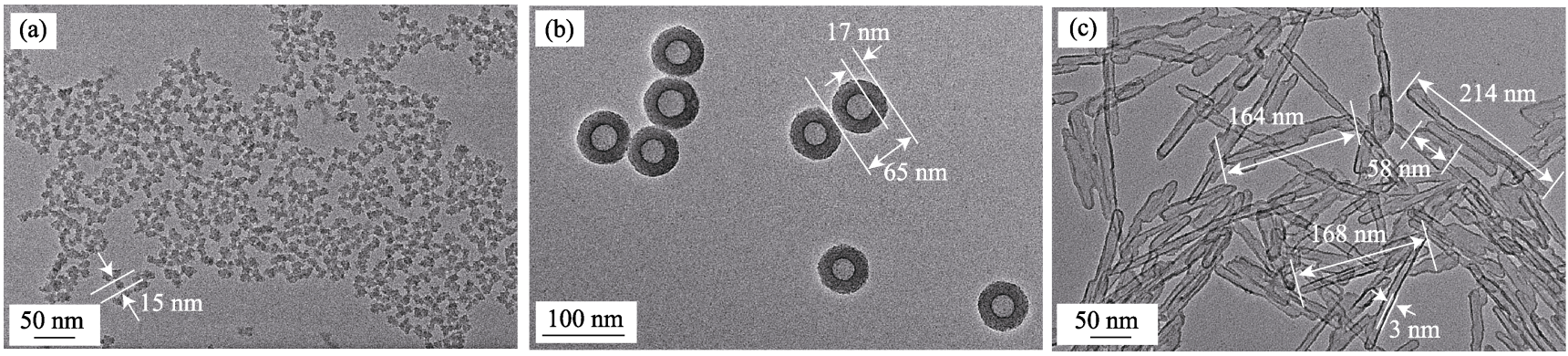

图 1 (a) $\mathrm{SiO}_{2}$ 粒子、 (b) $\mathrm{H}-\mathrm{SiO}_{2}$ 粒子和(c) $\mathrm{MgF}_{2}$ 棒高分辨透射电镜照片

Fig. 1 TEM images of (a) solid $\mathrm{SiO}_{2}$ particles, (b) $\mathrm{H}-\mathrm{SiO}_{2}$ particles and (c) hollow rod-shaped magnesium fluoride

均棒宽约 $15 \mathrm{~nm}$, 壁厚约 $3 \mathrm{~nm}$ (图 1(c))。

图 2 为镀制 $\mathrm{SiO}_{2} / \mathrm{H}-\mathrm{SiO}_{2} / \mathrm{MgF}_{2}$ 薄膜基片的场发 射扫描电镜照片, 薄膜具有由 $\mathrm{SiO}_{2}$ 和 $\mathrm{H}-\mathrm{SiO}_{2}$ 以及 $\mathrm{MgF}_{2}$ 粒子制备的单层膜叠加形成的梯度折射率膜 层结构。由椭偏仪测得的每层薄膜的厚度和折射率 如表 1 所示。 $\mathrm{MgF}_{2}$ 无规则地平铺在表面(图 2(a)), 棒 与棒之间有较大的空隙。观察到 $\mathrm{SiO}_{2}$ 实心粒子和 $\mathrm{H}-\mathrm{SiO}_{2}$ 空心粒子以及棒状 $\mathrm{MgF}_{2}$ 空心粒子在基片表 面上平铺紧凑、均匀, 层与层界限明显(图 2(b))。从 图中可得第一层 $\mathrm{SiO}_{2}$ 薄膜膜厚约为 $110 \mathrm{~nm}$, 第二层 $\mathrm{H}-\mathrm{SiO}_{2}$ 薄膜膜厚约为 $125 \mathrm{~nm}$, 第三层空心 $\mathrm{MgF}_{2}$ 薄 膜膜厚约为 $135 \mathrm{~nm}$, 与椭偏仪测试结果吻合。

使用酸催化 TEOS 得到的线性 $\mathrm{SiO}_{2}$ 溶胶不仅可 以调整膜层的折射率，而且能提高膜层的耐磨性 ${ }^{[16]}$, 顶层的氟化镁棒膜层又平铺得比较紧凑, 根据 ISO

表 $1 \mathrm{SiO}_{2} 、 \mathrm{H}-\mathrm{SiO}_{2} 、 \mathrm{MgF}_{2}$ 膜层椭偏仪测试结果

Table 1 Ellipsometer test results of $\mathrm{SiO}_{2}$, $\mathrm{H}-\mathrm{SiO}_{2}, \mathrm{MgF}_{2}$ films

\begin{tabular}{cccc}
\hline Layer position & Material & Thickness $/ \mathrm{nm}$ & $n_{(\lambda=550 \mathrm{~nm})}^{1}$ \\
\hline Layer 1 & $\mathrm{SiO}_{2}$ & 110 & 1.36 \\
Layer 2 & $\mathrm{H}^{-\mathrm{SiO}_{2}}$ & 125 & 1.25 \\
Layer 3 & $\mathrm{MgF}_{2}$ & 135 & 1.14 \\
\hline
\end{tabular}

1: refractive index
1518 标准, $\mathrm{SiO}_{2} / \mathrm{H}-\mathrm{SiO}_{2} / \mathrm{MgF}_{2}$ 薄膜经铅笔硬度测试 仪测试的硬度达到 4 5 H。采用划痕试验法测定薄 膜与基片的界面结合力，声发射峰出现波动的起始 点所对应的载荷值即为薄膜从基体脱落的临界载 荷 ${ }^{[17]}$, 实验测得 $\mathrm{SiO}_{2} / \mathrm{H}-\mathrm{SiO}_{2} / \mathrm{MgF}_{2}$ 梯度折射率薄膜 的临界载荷为 $25.8 \mathrm{~N}$ 。综合结果表明, 薄膜具有较 好的耐刮擦性及附着力。

\section{$2.2 \mathrm{SiO}_{2} / \mathrm{H}_{-}-\mathrm{SiO}_{2} / \mathrm{MgF}_{2}$ 梯度折射率薄膜光学} 性质

光垂直入射时(图 3(a)), 基片在 380 1600 nm 范 围的透光率随低折射率膜层逐渐镀制而逐渐升高。 空白基片最大透光率为 $91.75 \%$ 。镀制 $\mathrm{SiO}_{2}$ 单层膜 时, 最大透光率为 $98.06 \%$ 。镀制 $\mathrm{SiO}_{2} / \mathrm{H}-\mathrm{SiO}_{2}$ 双层 膜时, 显示出宽带减反膜效果, 最高透光率达到 $99.04 \%$ 。而镀制三层 $\mathrm{SiO}_{2} / \mathrm{H}-\mathrm{SiO}_{2} / \mathrm{MgF}_{2}$ 梯度折射率 薄膜时，最大透光率值高达 $99.88 \%$, 且平均透光率 达到 $98.93 \%$ ，显示出非常好的宽带减反射效果。

镀制 $\mathrm{SiO}_{2} / \mathrm{H}-\mathrm{SiO}_{2} / \mathrm{MgF}_{2}$ 薄膜基片在光不同入射 角下的透光率曲线图和三维图如图 3(b)和图 3(c) 所示。在 380 1600 $\mathrm{nm}$ 波段, 光在 $45^{\circ}$ 入射时最高 透光率值保持在 $98.84 \%$ 以上, 平均透光率则高于 $97.85 \%$ ，相比于垂直入射，仅下降了 $1.08 \%$ 。即使光 以 $75^{\circ}$ 入射, 最高透光率值仍达到 $95.51 \%$, 且平均
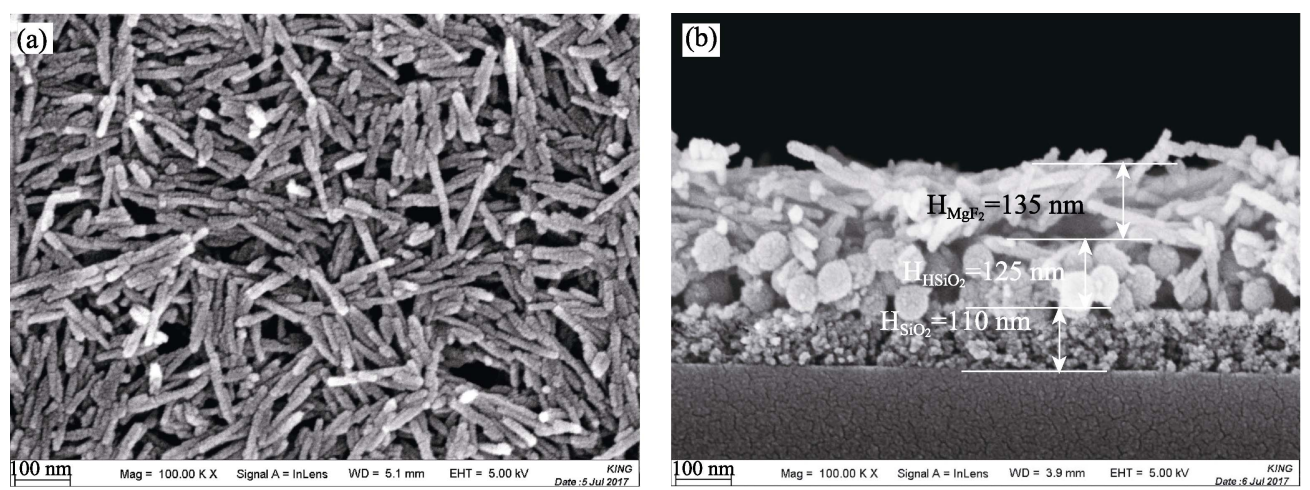

图 $2 \mathrm{SiO}_{2} / \mathrm{H}_{-} \mathrm{SiO}_{2} / \mathrm{MgF}_{2}$ 薄膜 FESEM (a)表面 SEM 照片和(b)截面

Fig. 2 FESEM (a) surface image, and (b) cross-sectional image of the $\mathrm{SiO}_{2} / \mathrm{H}_{-}-\mathrm{SiO}_{2} / \mathrm{MgF}_{2}$ film 

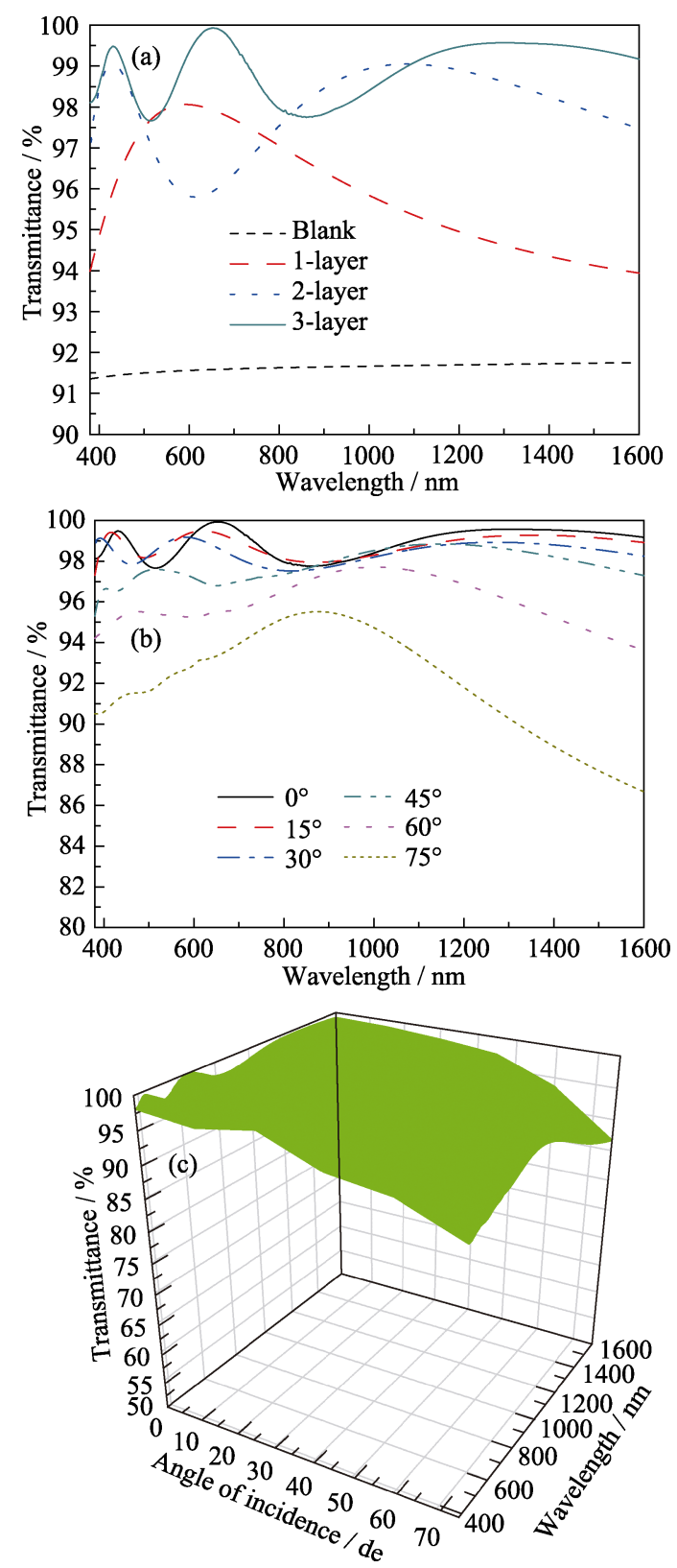

图 3 (a)镀 $\mathrm{SiO}_{2}$ 单层膜、 $\mathrm{SiO}_{2} / \mathrm{H}-\mathrm{SiO}_{2}$ 双层膜、 $\mathrm{SiO}_{2} / \mathrm{H}-$ $\mathrm{SiO}_{2} / \mathrm{MgF}_{2}$ 三层薄膜基片和空白基片在 380 1600 nm 波段内 的透光率曲线图, (b) $\mathrm{SiO}_{2} / \mathrm{H}-\mathrm{SiO}_{2} / \mathrm{MgF}_{2}$ 镀膜基片在不同光 入射角度下的透光率曲线图, (c) $\mathrm{SiO}_{2} / \mathrm{H}-\mathrm{SiO}_{2} / \mathrm{MgF}_{2}$ 镀膜基片 在不同入射角和不同波长下的透光率曲线三维图

Fig. 3 Transmittances of (a) the blank substrate and the coated substrates with $\mathrm{SiO}_{2}$ monolayer, $\mathrm{SiO}_{2} / \mathrm{H}-\mathrm{SiO}_{2}$ bilayer, and $\mathrm{SiO}_{2} / \mathrm{H}-\mathrm{SiO}_{2} / \mathrm{MgF}_{2}$ three-layer graded refractive index film in the wavelength of $380-1600 \mathrm{~nm}$, (b) the coated substrate with $\mathrm{SiO}_{2} / \mathrm{H}-\mathrm{SiO}_{2} / \mathrm{MgF}_{2}$ film at different angles of incidence, and (c) three-dimensional transmittances of the coated substrate with $\mathrm{SiO}_{2} / \mathrm{H}-\mathrm{SiO}_{2} / \mathrm{MgF}_{2}$ film

透光率也到达 $92.05 \%$ 。

在大角度入射下，基片的透光率下降是因为光 倾斜入射时, 折射率被分离成 $s$-偏振光和 $p$-偏振光 的两个有效折射率, 使界面处的菲涅耳系数显著增 大，从而界面反射率随着倾斜角度的增加而增大 ${ }^{[18]}$ 。
而镀膜基片可以在大角度宽波段下达到良好的减反 射效果, 是由于梯度折射率薄膜的菲涅尔反射系数 很小 ${ }^{[5,19]}$ 。根据光学薄膜理论 ${ }^{[20]}$, 多层均质膜光学特 性通过以下导纳矩阵进行计算 ${ }^{[21]}$ :

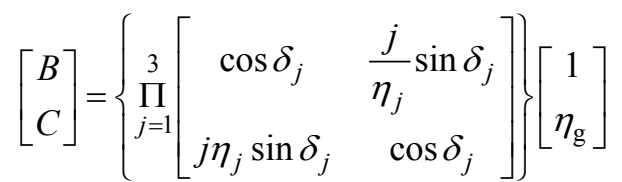

则多层膜系的理论透光率值为:

$$
T=1-R(\lambda)=1-\left(\frac{n_{0} B-C}{n_{0} B+C}\right)\left(\frac{n_{0} B-C}{n_{0} B+C}\right)^{*}
$$

而 $p$-偏振光和 $s$-偏振光的有效光学厚度都为:

$$
\delta_{j}=\frac{2 \pi}{\lambda} n_{j} d_{j} \cos \theta_{j}
$$

对应的 $s$-偏振光和 $p$-偏振光的有效折射率为:

$$
\eta_{j}= \begin{cases}n_{j} \cos \theta_{j} & s-\text { 偏振 } \\ \frac{n_{j}}{\cos \theta_{j}} & p-\text { 偏振 }\end{cases}
$$

则在 $z$ 和 $z+\mathrm{d} z$ 之间的界面处如图 4(a)所示, 菲涅耳 反射系数可以表示为 ${ }^{[18]}$ :

$$
r_{s}=\left(\frac{1}{2 \eta_{s}(\mathrm{z})}\right) \frac{\mathrm{d} \eta_{s}(z)}{\mathrm{d} z}=\frac{n^{\prime}(z)}{2 n(z)}-\frac{\theta^{\prime}(z)}{2} \tan \theta(z)
$$

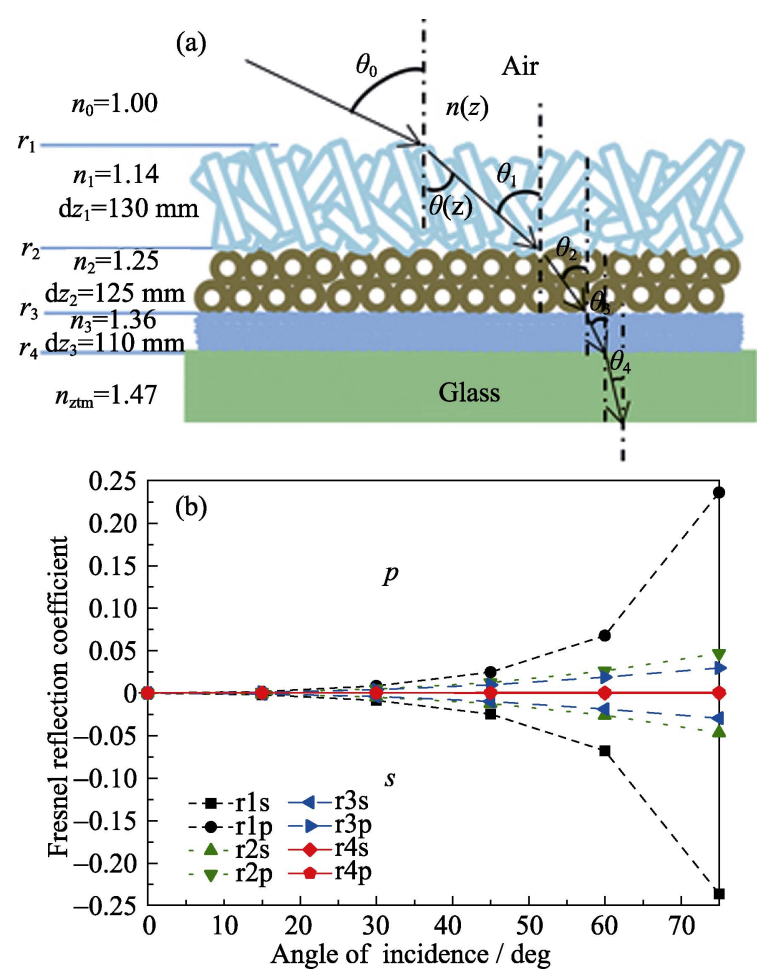

图 4 (a)光通过三层薄膜进入基底的光路图, (b)不同入射角 下膜层界面处的菲涅耳反射系数

Fig. 4 (a) Optical path of the light enters in the substrate through three-layer coating, (b) Fresnel reflection coefficients at the coating interfaces at different angles of incidence 
和

$$
r_{p}=\left(\frac{1}{2 \eta_{p}(\mathrm{z})}\right) \frac{\mathrm{d} \eta_{\mathrm{p}}(z)}{\mathrm{d} z}=\frac{n^{\prime}(z)}{2 n(z)}+\frac{\theta^{\prime}(z)}{2} \tan \theta(z)
$$

其中 $B$ 和 $C$ 分别是在介质中传播的光的总电场和磁 场幅度, $n_{0}$ 为空气的折射率, $\theta(z), \eta_{\mathrm{g}}, n_{j}(j=1,2,3)$ 和 $d_{j}(j=1,2,3)$ 是光的入射角度、玻璃基底的折 射率以及第 $j$ 层薄膜的折射率和物理厚度。结合 式(3) (6), 光以不同入射角度通过空气穿过膜层到 达基底之间的界面处的菲涅尔反射系数如图 4(b)所 示, 从图中可以看出, 菲涅耳反射系数在 $0^{\circ}$ 入射下 不会分离, 而当入射角从 $0^{\circ}$ 增加到 $75^{\circ}$ 时, 同一界 面处的菲涅尔反射系数逐渐发生分离, 但菲涅尔反 射系数从 $r_{1}$ 到 $r_{4}$ 的分离在逐渐减小。并且, 同一界 面的 $r_{s}$ 和 $r_{p}$ 值保持在一个较小的范围内分离, 这也 是镀膜基片能实现全向宽带减反射效果的原因。因 此, 如果菲涅尔反射系数的分离能进一步减小, 则 可获得更大角度的宽带抗反射效果 ${ }^{[19]}$ 。

镀制 $\mathrm{SiO}_{2} / \mathrm{H}-\mathrm{SiO}_{2} / \mathrm{MgF}_{2}$ 三层梯度折射率薄膜基 片在 380 1600 nm 波段内，以不同角度光入射的理 论透光率和实验非偏振测量透光率和偏振透光率曲 线如图 5 所示, 其理论透射率略大于测量(非偏振) 透射率，这可能是由于薄膜是溶胶浸渍提拉而成， 薄膜在提拉镀制过程中, 基片双面薄层的厚度和孔
隙率都会出现少许差异, 进而导致各层的折射率也 会出现轻微的偏差, 且薄膜的顶层为无规则棒状氟 化镁构建而成, 其表面较大的粗粘度(图 2)引起薄 膜表面入射光的散射，从而使实验透射率与理论透 射率有所偏差 ${ }^{[22]}$, 但是透光率峰值出现的位置吻合 度很高, 差异非常小。光垂直入射时(图 5(a)), 透射 率在 433、658 和 $1323 \mathrm{~nm}$ 的波长处具有极大值, 分 别为 $99.47 \% 、 99.88 \%$ 和 $99.60 \%$ 。光入射角为 $30^{\circ}$ 时(图 5(b)), 非偏振和偏振透过率都保持在 99\%以 上。光入射角为 $60^{\circ}$ 时(图 5(c)), 非偏振最大透射率 仍达到 $97.70 \%$ 。光入射角为 $75^{\circ}$ 时(图 5(d)), 最大非 偏振透过率仍达到 $95.51 \%$ 。同时还看出, 镀膜基片 的偏振透射率分离程度较小。光入射角为 $30^{\circ}$ 时, $p$-和 $s$-偏振透过率在宽波长范围内均高达 $99 \%$, 接近于 垂直入射的透过率。而光入射角为 $60^{\circ}$ 时, $p$-和 $s$-偏 振透过率均高于 $95 \%$, 分离程度在 $1 \% \sim 5 \%$ 之内。对 于 $75^{\circ}$ 的高入射角光，镀膜基片的高抗反射效率也是 显著的, 其 $p$-和 $s$-偏振透过率分离程度也不超过 $8 \%$ 。

\section{$2.3 \mathrm{SiO}_{2} / \mathrm{H}_{-}-\mathrm{SiO}_{2} / \mathrm{MgF}_{2}$ 梯度折射率薄膜的自} 清洁疏水性能

接触角测试结果表明, 未改性的 $\mathrm{SiO}_{2} / \mathrm{H}-\mathrm{SiO}_{2} /$ $\mathrm{MgF}_{2}$ 薄膜的接触角为 $121.8^{\circ}$ (图 6(a)上), 其 $\mathrm{AFM}$ 结 果(图 6(a)下)表明膜层的表面粗粘度 $R_{\mathrm{q}}$ 为 $22.8 \mathrm{~nm}$,
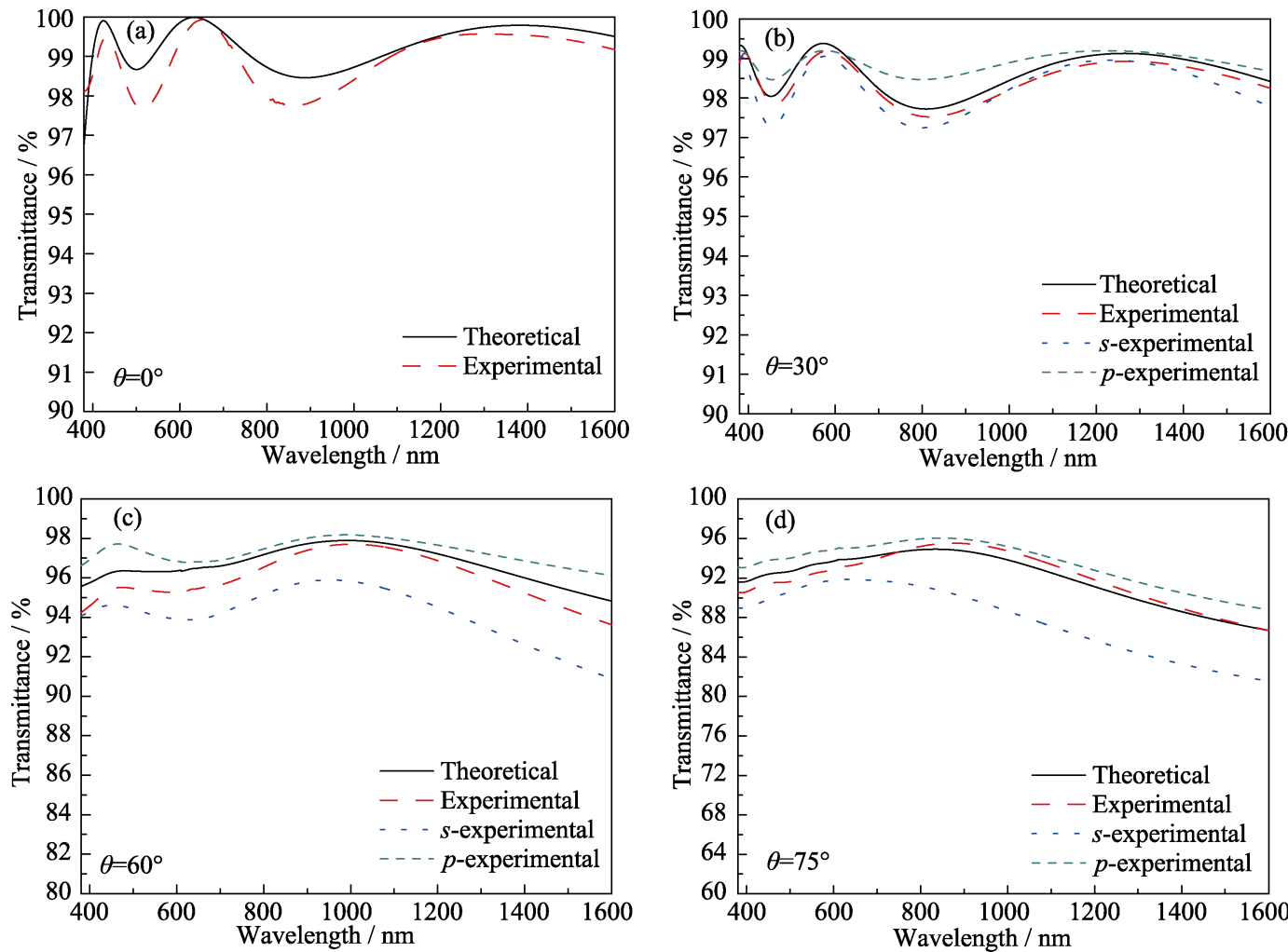

图 $5 \mathrm{SiO}_{2} / \mathrm{H}_{-}-\mathrm{SiO}_{2} / \mathrm{MgF}_{2}$ 梯度折射率薄膜在(a) $0^{\circ}$ 、 (b) $30^{\circ} 、$ (c) $60^{\circ}$ 和(d) $75^{\circ}$ 的光入射角度下的理论和实验非偏振和偏振透光率曲线 Fig. 5 Theoretical and experimental and polarization transmittance curves of the $\mathrm{SiO}_{2} / \mathrm{H}-\mathrm{SiO}_{2} / \mathrm{MgF}_{2}$ three-layer graded refractive index film coated substrate at the incident angles of (a) $0^{\circ}$, (b) $30^{\circ}$, (c) $60^{\circ}$, (d) $75^{\circ}$ 


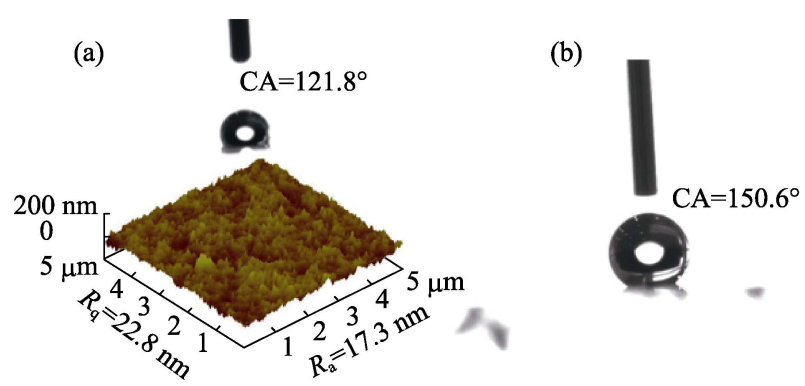

图 6 (a) $\mathrm{SiO}_{2} / \mathrm{H}-\mathrm{SiO}_{2} / \mathrm{MgF}_{2}$ 梯度折射率薄膜修饰前的水接 触角和 AFM 图, (b) HDTMOS 修饰后的水接触角

Fig. 6 (a) Water contact angle and AFM pattern of the $\mathrm{SiO}_{2} / \mathrm{H}-\mathrm{SiO}_{2} / \mathrm{MgF}_{2}$ gradient index film, and (b) water contact angle of the $\mathrm{SiO}_{2} / \mathrm{H}-\mathrm{SiO}_{2} / \mathrm{MgF}_{2}$ film was modificated by HDTMOS

$R_{\mathrm{a}}$ 为 $17.3 \mathrm{~nm}$ 。没有经过处理的薄膜表面具有如此 大的疏水角度, 主要是由于 $\mathrm{SiO}_{2} / \mathrm{H}-\mathrm{SiO}_{2} / \mathrm{MgF}_{2}$ 薄膜 表面的棒状 $\mathrm{MgF}_{2}$ 形成了结构粗䊁的薄膜表面, 图 2(b)也证明薄膜表面有许多棒状氟化镁凸起，从 而使薄膜表面具有一定的粗䊁度。当使用 HDTMOS 进行表面改性后，其接触角增加至 150.6²(图 6(b)), 薄膜将表现出良好的疏水自清洁效果。

\section{3 结论}

研究利用二氧化硅实心粒子和空心二氧化硅颗 粒以及空心氟化镁纳米棒成功制备出三层折射率逐 渐变化的薄膜，在宽波段和大角度下显著提高了薄 膜的透射率，显示出良好的全向宽带抗反射性能。另 外, 由于使用棒状氟化镁制备第三层薄膜, 其无规 则的堆积在表面形成了合适的粗䊁度，使用 HDTMOS 修饰后, 薄膜具有了超疏水自清洁效果, 因此实现了减反膜宽谱带、宽角度减反射和超疏水 自清洁的多功能性能。

\section{参考文献:}

[1] GREEN MARTIN A, EMERY KEITH, HISHIKAWA YOSHIHIRO, et al. Solar cell efficiency tables (version 45). Progress in Photovoltaics: Research and Applications, 2015, 23(1): 1-9.

[2] BARAN DERYA, ASHRAF RAJA SHAHID, HANIFI DAVID A, et al. Reducing the efficiency-stability-cost gap of organic photovoltaics with highly efficient and stable small molecule acceptor ternary solar cells. Nature Materials, 2017, 16(3): 363-369.

[3] CHHAJED SAMEER, SCHUBERT MARTIN F, KIM JONG $\mathrm{KYU}$, et al. Nanostructured multilayer graded-index antireflection coating for Si solar cells with broadband and omnidirectional characteristics. Applied Physics Letters, 2008, 93(25): 251108-1-3.

[4] LIU BO-TAU, YEH WEI-DE. Antireflective surface fabricated from colloidal silica nanoparticles. Colloids and Surfaces A: Physicochemical and Engineering Aspects, 2010, 356(1): 145-149.
[5] CHATTOPADHYAY S, HUANG Y F, JEN Y J, et al. Antireflecting and photonic nanostructures. Materials Science and Engineering: R: Reports, 2010, 69(1): 1-35.

[6] RAUT HEMANT KUMAR, GANESH V ANAND, NAIR A SREEKUMARAN, et al. Anti-reflective coatings: a critical, in-depth review. Energy \& Environmental Science, 2011, 4(10): 3779-3804.

[7] CAMARGO KeLly C, MiCHELS ALEXANDRE F, RODEMBUSCH FABIANO S, et al. Multi-scale structured, superhydrophobic and wide-angle, antireflective coating in the near-infrared region. Chemical Communications, 2012, 48(41): 4992-4994.

[8] LIU LI-QIANG, WANG XIAO-LIN, JING MIN, et al. Broadband and omnidirectional, nearly zero reflective photovoltaic glass. $A d-$ vanced Materials, 2012, 24(47): 6318-6322.

[9] YILDIRIM ADEM, KHUDIYEV TURAL, DAGLAR BIHTER, et al. Superhydrophobic and omnidirectional antireflective surfaces from nanostructured ormosil colloids. ACS Applied Materials \& Interfaces, 2013, 5(3): 853-860.

[10] YOLDAS BULENT E. Investigations of porous oxides as an antireflective coating for glass surfaces. Applied Optics, 1980, 19(9): $1425-1429$.

[11] STÖBER WERNER, FINK ARTHUR, BOHN ERNST. Controlled growth of monodisperse silica spheres in the micron size range. Journal of Colloid and Interface Science, 1968, 26(1): 62-69.

[12] WAN YONG, YU SHU-HONG. Polyelectrolyte controlled largescale synthesis of hollow silica spheres with tunable sizes and wall thicknesses. The Journal of Physical Chemistry C, 2008, 112(10): 3641-3647.

[13] JI ZIHAN, BAO LEI, WANG HONGNING, et al. Preparation of super-hydrophobic antireflective films by rod-like $\mathrm{MgF}_{2}$ and $\mathrm{SiO}_{2}$ mixed sol. Materials Letters, 2017, 207: 21-24.

[14] THOMAS LAN M. Method for the preparation of porous silica antireflection coatings varying in refractive index from 1.22 to 1.44 . Applied optics, 1992, 31(28): 6145-6149.

[15] WANG Y, HE M Y, CHEN R Y. Fabrication of mechanically robust antireflective films using silica nanoparticles with enhanced surface hydroxyl groups. Journal of Materials Chemistry A, 2015, 3(4): 1609-1618.

[16] YE HAIPING, ZHANG XINXIANG, ZHANG YULU, et al. Preparation of antireflective coatings with high transmittance and enhanced abrasion-resistance by a base/acid two-step catalyzed Sol-Gel process. Solar Energy Materials and Solar Cells, 2011, 95(8): 2347-2351.

[17] WANG JIGANG, LIU MIN. Study on the tribological properties of hard films deposited on biomedical NiTi alloy. Materials Chemistry and Physics, 2011, 129(1/2): 40-45.

[18] POITRAS D, DOBROWOLSKI J A. Toward perfect antireflection coatings. 2. Theory. Applied Optics, 2004, 43(6): 1286-1295.

[19] XI J Q, SCHUBERT M F, KIM J K, et al. Optical thin-film materials with low refractive index for broadband elimination of Fresnel reflection. Nature Photonics, 2007, 1(3): 176-179.

[20] REUNA JARNO. Anti-reflective Coatings for Multi-junction Solar Cells. Finland: Master's Science Thesis of Tampere University of Technology, 2016.

[21] DUYAR ÖZLEM, DURUSOY HÜSEYIN ZAFER. Design and preparation of antireflection and reflection optical coatings. Turkish Journal of Physics, 2004, 28(2): 139-144.

[22] BAO LEI, WU JIAN, WANG HONGNING, et al. Design and preparation of broadband antireflective coatings with excellent mechanical properties. Materials Letters, 2016, 185: 464-467. 